\title{
E1A and the Ewing tumor translocation
}

To the editor-In the September issue of Nature Medicine, Sanchez-Prieto et al. ${ }^{1}$ reported that the adenoviral E1A protein induces the EWS-FLI1 rearrangement specific to Ewing tumor ${ }^{2}$ in a variety of human cells. These results led to the proposal that EWS-FLI1 expression may be important for the transforming properties of E1A, a protein already known to interfere with cellular pathways relevant to oncogenesis, such as those controlled by p53 and Rb. They also raised the revolutionary hypothesis of the Ewing tumor being of viral origin.

We have attempted to reproduce these results using HEK and HEK293 cells from various sources. Surprisingly, although HEK293 cells, in contrast to parental HEK cells, express high levels of both 289R and 243R isoforms of E1A, the EWS-FLI1 protein could not be detected in any of these cell lines. Moreover, the highly sensitive RT-PCR method did not detect an EWS-FLI1 fusion transcript.

We also note discrepancies in the figures presented by the authors. In Fig. 1 (ref. 1), the established HEK293 cell line expresses a type 1 EWS-FLI1 fusion transcript. Conversely, Fig. $2 c$ and d indicate that this same cell line expresses a protein and a fusion transcript of the same molecular weight as that of the RDES cell line known to have an EWS-FLI1 type 2 RNA and protein ${ }^{3}$. The authors claim that the different sizes of the EWS-FLI1 proteins or cDNAs found throughout the manuscript are linked to different retroviral infections and correlated with the variety of fusion transcripts found in Ewing tumors ${ }^{4}$. Although this explanation may be relevant for the results obtained with retrovirally infected IMR90 cells, it does not account for the established HEK293 cell line.

Being familiar with fluorescence in situ hybridization of Ewing cells and having provided the authors with the $\cos 1 \mathrm{D} 1$ and cosG9 cosmids $^{5}$ (as mentioned in their acknowledgments), we believe that the results of Fig. 3 are inconsistent with a $\mathrm{t}(11 ; 22)$ translocation. In Fig. $3 a$, only one derivative chromosome is seen, whereas two would be expected. More strikingly, Fig. $3 b$ shows a chromosome hybridizing with both a centromeric chromosome 11 probe and the cosG9 chromosome 22 cosmid; this is therefore suspected to be a der(11) chromosome. However, as the cosG9 cosmid lies proximal to the translo- cation breakpoint region on chromosome 22 , it is always localized on the $\operatorname{der}(22)$ and never on the der(11) chromosome in the case of a Ewing $t(11 ; 22)$ translocation.

We cannot rule out the possibility that some E1A-expressing IMR90 or HeLa cells may occasionally present an EWS-FLI1 fusion, as we have not studied these cells. However, we would like to emphasize that the presence of an EWS-FLI1 fusion is not a characteristic of the type 5 adenovirustransformed HEK293 cell line. We can therefore conclude that the generation of the $t(11 ; 22)$ translocation does not necessarily follow transformation with E1A.

\section{Thomas Melot \& Olivier Delattre \\ Lab. de Pathologie Moleculaire des Cancers INSERM U509, Institut Curie \\ 26 rue d'Ulm \\ 75248 Paris, France}

To the editor-Breakpoint variation and the resulting fusion type heterogeneity are characteristic of the Ewing tumor-associated EWS-FLI1 gene rearrangement. Sanchez-Prieto et al. report high efficiency induction of EWS-FLI1 recombination in a variety of cell types by adenovirus E1A (ref 1 ). To our surprise, only a single fusion transcript type (type 1 or type 2 ) was found in individual E1A transfections, instead of the expected polyclonal rearrangement. To characterize the E1A-induced gene fusions in more detail, we sought to repeat the experiments described by Sanchez-Prieto et al.

Adenovirus 5 E1A was expressed from a CMV promoter in HeLa cervix carcinoma cells, a human fibroblast cell line and a neuroblastoma cell line after lipofection. Transfection efficiencies were approximately $2 \%, 4 \%$ and $80 \%$, respectively. We used a one-tube nested PCR approach capable of identifying one EWS-FLI1-expressing cell in a total of $10^{6}$ cells $^{6}$ to screen for expression of the Ewing tumor specific gene rearrangement 70 hours after transfection. No EWS-FLI1 transcript was detectable in any of the transiently E1A-transfected cells, although E1A expression was confirmed.

Next, we studied HEK293 human embryonal kidney cells immortalized with adenovirus 5 DNA and a subclone of this cell line EcR293 obtained from two different commercial sources (ATCC and Invitrogen), for which a common origin could be confirmed by microsatellite analysis. Although Sanchez-Prieto et al. reported that HEK293 cells carry and express an EWS-FLI1 type 1 rearrangement, we were unable to detect the presence of the gene rearrangement on Southern blot analysis of genomic DNA (a piece of evidence lacking from the study by SanchezPrieto et al.); nor could we identify expression of an EWS-FLI1 chimeric RNA by northern blot analysis, by RT-PCR using the conditions applied by the authors, or by single-tube nested PCR. Furthermore, the EWS-FLI1 protein was undetectable on western blots probed with an FLI1-specific polyclonal antibody ${ }^{7}$. In contrast, all Ewing tumor cell lines studied in parallel, tested positive for EWS-FLI1 using all the methods described herein.

Finally, we tested 27 Ewing tumor cell lines and 19 primary Ewing tumors with established EWS-FLI1 expression for the presence of adenovirus 5 E1A DNA/RNA by RT-PCR. None of the samples produced positive results.

In summary, we were unable to confirm an association between adenovirus E1A expression and the oncogenic EWS-FLI1 gene rearrangement, either in Ewing tumors or in any other cell type tested. Thus, there is no evidence for a major role of viral genes in the generation of Ewing tumors.

\section{HEINRICH KOVAR}

CCRI St. Anna Kinderspital

Kinderspitalgasse 6

A-1090 Vienna, Austria

Email:kovar@ccri.univie.ac.at

1 Sanchez-Prieto et al. An association between viral genes and human oncogenic alterations: The adenovirus E1A induces the Ewing tumor fusion transcript EWS-FLI1. Nature Med. 5, 1076-1079 (1999).

2 Delattre, O. et al. Gene fusion with an ETS domain caused by chromosome translocation in human tumors. Nature 359, 162-165 (1992).

3 Giovannini, M. et al. EWS-erg and EWS.FLI1 fusion transcripts in Ewing's sarcoma and primitive neuroectodermal tumours with variant translocations. J. Clin. Invest. 94, 489-496 (1994).

4 Zucman, J. et al. Combinatorial generation of variable fusion proteins in peripheral primitive neuroectodermal tumors. EMBO J. 12, 4481-4487 (1993).

5. Desmaze, $\mathrm{C}$ et al. Interphase molecular cytogenetics of Ewing's sarcoma and peripheral neuroepithelioma $\mathrm{t}(11,22)$ translocation with flanking and overlapping cosmid probes. Cancer Genet. Cytogenet. 74, 13-18 (1994).

6. Pete, M. et al. Sensitive detection of occult Ewing's cells by the reverse transcriptase-polymerase chain reaction. Br.J.Cancer 72, 96-100 (1995).

7. Melot, T. et al. Production and characterization of mouse monoclonal antibodies to wild-type and oncogenic FLI-1 proteins. Hybridoma 16, 457-464 (1997).

We will publish a response from Sanchez-Prieto et al. in the January 2000 issue....Ed. 